\title{
Os desafios da tradução em ferramentas de pesquisa: a questão cultural e o design da informação
}

\author{
Challenges in translating research tools: cultural aspects and information design
}

Claudia Mont'Alvão, Carolina Esteves, Mariana Furtado Dias

Ferramentas de pesquisa, linguagem simples, comportamento, aspectos culturais.

É comum nas pesquisas e projetos em Design a utilização de escalas, formulários, questionários, listas de verificação dentre outras ferramentas de coleta de dados utilizadas propostas por pesquisadores, em outro idioma que não o português. Esta dinâmica permite uma construção contínua do conhecimento e melhor compreensão de sua aplicação além da validação dessas ferramentas. No entanto, esse procedimento inclui a versão dessas ferramentas para o idioma português pelos pesquisadores que vão utilizá-las. Nesse contexto, esse artigo apresenta dois exemplos dessa prática e as diferenças que podem ser encontradas nesse procedimento de tradução. A partir dessa discussão, são destacados os aspectos culturais e também a questão do design da informação, que se tornam desafios nessa prática. São apontadas sugestões para que novas traduções sejam realizadas, visando um equilíbrio e melhor difusão das ferramentas já traduzidas para o Português.

Research tools, translation Plain language, behaviour, cross-cultural aspects.

It is common in research and Design projects to use scales, forms, questionnaires, checklists, among other data collection tools used for research that are in a language other than Portuguese. This dynamic allows continuous improvement and enhances a better comprehension of these tools and their validation. Nevertheless, this procedure includes the translation of these tools into Portuguese by the researchers that will use them. In this context, this paper presents two examples of this practice and the differences that can be found in this translation procedure. This discussion highlighted the cross cultural aspects and the information design approach as challenges of this practice. Suggestions are also proposed for the new translations aiming at equilibrium among cultures and better dissemination of tools already translated to Portuguese.

\section{Introdução}

A expansão da pesquisa no Brasil é evidente. Em 2020 dezenove pesquisadores brasileiros foram citados ao integrarem a lista "Pesquisadores Altamente Citados 2020", pelo site Web of Science (2020), da empresa Clarivate Analytics. A citação desses pesquisadores por outros colegas da mesma área demonstra a sua influência em nível mundial. Segundo o ranking apresentado, 6.389 pesquisadores de mais de 60 países foram incluídos na lista de 2020 . Desses, 3.896 apresentaram produção científica com impacto em áreas específicas e 2.493 com impacto cruzado, ou seja, em diversos campos do conhecimento.

Anais do $10^{\circ} \mathrm{CIDI}$ e $10^{\circ} \mathrm{CONGIC}$

Kelli C.A.S. Smythe, Rafael de Castro Andrade (orgs.)

Sociedade Brasileira de Design da Informação - SBDI

Curitiba | Brasil | 2021
Proceedings of the $10^{\text {th }} \mathrm{CIDI}$ and $10^{\text {th }} \mathrm{CONGIC}$

Kelli C.A.S. Smythe, Rafael de Castro Andrade (orgs.)

Sociedade Brasileira de Design da Informação - SBDI Curitiba | Brazil | 2021 
Quando tratamos a pesquisa em Design, o cenário não é muito diferente. A área conta em 2021 com 22 Instituições credenciadas que oferecem Programas de Pós-Graduação, em nível de Mestrado e Doutorado. Destacam-se aí os cursos com notas 6 e 5 segundo a última avaliação da CAPES (2017). Nesses Programas de Pós-Graduação, tem-se uma produção consistente de docentes e discentes não somente m português, mas também em outros idiomas.

Entre teses, dissertações, projetos patrocinados e pesquisas em Iniciação Científica, é frequente o uso de entrevistas, questionários, listas de verificação, escalas de diferencial semântico, entre outras ferramentas mais especificas. Pode-se aqui destacar o uso de escalas de diferencial semântico nas pesquisas de experiência do usuário e as relações do design e emoções provocadas por produtos e sistemas.

Penteado e Ribeiro (2016) mencionam que 'o estudo sobre ferramentas que facilitam a compreensão das emoções provindas da relação indivíduo/objeto contribuem para a aplicação do Design Emocional na criação de produtos que atinjam de maneira efetiva seu público alvo'.

Quando tratamos dessas ferramentas de pesquisa, e também de seu relato (como esse artigo, dissertações, teses, relatório) podemos entender a necessidade se clareza e objetividade nessas informações a partir da definição de Pettersson (2002) que afirma:

Do ponto de vista terminológico o conceito de 'informação pode ser apresentado entre os conceitos de 'dado' e 'conhecimento'. Não é fácil delinear um limite entre esses conceitos. Nós podemos compreender a informação a partir de várias perspectivas a respeito de como criamos, apresentamos, produzimos, distribuímos, pesquisamos, classificamos, indexamos, armazenamos, recebemos, processamos, valoramos, respondemos, usamos e renovamos a informação.

Aliado ao conceito de informação e projeto de informação, tem-se o conceito de 'linguagem simples'. Redish (2000), em seu livro What is information design? menciona que a linguagem simples busca criar comunicações que permitem as pessoas "a encontrar o que elas precisam, compreender o que elas procuram e utilizar o que elas compreendem apropriadamente".

Essa afirmativa de Redish foi elaborada pelos grupos de linguagem simples internacionais e do Governo americano, e a PLAIN (Plain Language Association International, 2021) apresenta a proposta de definição da Federação Internacional de Linguagem Simples que diz ${ }^{1}$ :

Uma comunicação está em linguagem simples se sua escrita, estrutura e design são tão claros que o público alvo consegue identificar facilmente o que procura, compreender o que encontrou e usar essa informação o que eles e utilizam aquela informação.

Schriver (2017, p. 345) comenta que linguagem clara pode ser um processo e um produto:

O campo da linguagem clara diz respeito ao processo de criação de comunicações amigáveis para as pessoas e aos produtos resultantes. O processo envolve adequar 0 conteúdo de forma iterativa, com cada versão sendo refinando a comunicação sucessivamente

\footnotetext{
${ }^{1}$ Vale observar na aba do site na versão em português está escrito 'Linguagem clara'. Esse é o termo adotado em Portugal, e no Brasil, desde 2015. Contudo, a partir de 2020, no Brasil, nos documentos oficiais esse termo foi substituído por 'Linguagem Simples'. Tal mudança deveu-se ao entendimento de conotação negativa da palavra claro (em oposição à 'escuro'). Desta forma o artigo utiliza a terminologia adotada no Brasil. Também é possível encontrar documentos da área de inovação e governança adotando o tempo 'Linguagem cidadã'.
} 
visando o melhor atendimento às necessidades das pessoas. Como outros profissionais de comunicação, os defensores da linguagem simples baseiam-se em sua experiência em comunicação e no feedback daqueles que podem ter interesse em seu trabalho (leitores, telespectadores, ouvintes, usuários, chefes, colegas e advogados).

Schriver (2017, p. 346), completa ainda que "assim como os profissionais de design da informação, os profissionais de linguagem clara dispendem bom tempo pensando em estratégias para orquestrarem palavras, imagens e tipografia".

No Brasil, as diretrizes de linguagem simples datam de 2020 (Brasil, 2020). As diretrizes nacionais seguem aquelas já estabelecidas por governos locais - como do Governo do Estado do Ceará e da Prefeitura Municipal de São Paulo -, além de outros países que consideram que a linguagem simples deve ser utilizada em todas as formas de comunicação.

Diante desse cenário, e correlacionando o conceito de informação de Pettersson e Schriver apresentam-se as seguintes questões: como os pesquisadores - com um recorte na área do Design - estão realizando as traduções dessas ferramentas? Será que a versão proposta realmente apresenta os aspectos do idioma nos quais foram concebidos? São informações claras e bem compreendidas? De que forma essa versão é entendida pelos respondentes?

É a partir dessas questões que se desenvolve o presente texto. Partindo da fundamentação teórica sobre o tema, esse artigo apresenta dois exemplos dessa prática e as diferenças que podem ser encontradas nesse procedimento de tradução/ versão.

\section{Um pouco sobre tradução}

Para fundamentação da pesquisa, considerando ser este texto produzido e discutido no campo do Design, é importante apresentar o que se entende por tradução.

Segundo de Sousa (1998, p. 51), o termo tradução pode significar "o produto (ou seja, o texto traduzido; (b) o processo do ato tradutório; (c) o ofício (a atividade de traduzir); ou (d) a disciplina (o estudo interdisciplinar e/ou autônomo). Segundo o autor, são diversas as posturas teóricas acerca de uma conceituação. Completa, ainda, citando que "a controvérsia mais antiga em torno da tradução diz respeito à tensão entre tradução literal e livre, duas posições frontalmente opostas. Ao conceito de tradução literal está associada a ideia de tradução fiel, neutra, objetiva, e ao de tradução livre, a ideia de tradução infiel, parcial, subjetiva"

Toda língua é uma estrutura formal (um código) que consiste em elementos que podem combinar-se para veicular 'sentido' semântico. Ao mesmo tempo, a língua é um sistema de comunicação que emprega as formas do código para referir-se a entidades e cria sinais que possuem 'valor' comunicativo, conforme Bell (1991, p. 6). Continuando essa discussão, Bell (1991, p. 7) afirma que no processo de tradução, o tradutor tem opção de focalizar nas equivalências formais, "que 'preservam' o sentido semântico do texto original", ou nas equivalências funcionais, "que 'preservam' o valor comunicativo do texto".

O conceito mais comum de tradução, segundo os linguistas, não é o de "transferência", mas o de "substituição" de significados (Catford, 1980, p. 53). O autor cita que: 
Por meio dos exemplos como os precedentes deveria ficar claro que é possível uma espécie restrita de "transferência de significado" de uma língua para outra; mas fica igualmente claro que isso não é o que normalmente se entende por tradução. Em "tradução" há substituição de significados da LF [Língua-Fonte] por significados da LM [Língua-Meta]: não transferência de significados da LF para a LM. Na transferência há uma implantação de significados da LF no texto da LM. Esses dois processos devem ser claramente diferenciados em qualquer teoria de tradução.

Tratando o tema no contexto da informação, Candell et al. (1986) afirmam que a alta fidelidade de instrumentos de medição de um idioma para outro é necessária para comparações significativas entre e dentro de culturas e grupos de idiomas.

Assim, entende-se que o processo de tradução das ferramentas que utilizamos na área do design, oriundas de outros idiomas não se constitui em um processo fácil, colocando em língua portuguesa o termo apresentado no idioma original.

Brislin (1970) sugere que ao traduzirmos uma ferramenta de pesquisa a partir da língua inglesa devem ser considerados três momentos distintos:

- primeiro, a versão original em inglês é traduzida para a língua alvo (no caso, português),

- então, essa versão traduzida é revisada por indivíduos bilíngues, que não leram a versão original em inglês, fazendo a tradução reversa, para o inglês,

- as versões original e traduzida são então comparadas e as discrepâncias são discutidas entre os pesquisadores envolvidos.

Uma vez entendida a necessidade de uma tradução apropriada dos instrumentos de pesquisa, apresentam-se, a seguir, exemplos desse processo.

\section{Metodologia}

A fim de ilustrar a importância e as diferenças de o processo de tradução pode ocasionar em instrumentos de pesquisa, são apresentados 2 exemplos.

No primeiro exemplo, apresenta-se a tradução da ferramenta AttrakDiff2 ${ }^{\mathrm{TM}} 2.0^{2}$. Originalmente proposta em alemão, sua tradução para o inglês é oferecida por seus autores. Encontram-se também suas traduções em trabalhos publicados em português, como será detalhado a seguir. Nesse primeiro exemplo utilizou-se a abordagem de tradução de painel de especialistas, que atende às premissas de Bell (1991).

No segundo exemplo, a tradução de um formulário em formato de questionário a partir da técnica de tradução proposta por Brislin (1970). Aqui, verificou-se que mesmo com o rigor do processo de tradução, é possível chegar a um documento que não atende aos requisitos de uma informação simples e compreensível, e por isso, foi adotada a abordagem da linguagem simples.

\footnotetext{
${ }^{2}$ AttrakDiff2 ${ }^{\mathrm{TM}}$ 2.0. www.attrakdiff.de
} 


\section{Exemplo 1: traduzindo a ferramenta AttrakDiff2 ${ }^{\mathrm{TM}}$}

O AttrakDiff2 ${ }^{\mathrm{TM}} 2.0^{3}$, foi proposto por Hassenzhal (2004) e avalia a percepção dos usuários sobre atributos de produtos pela combinação de aspectos do produto (p.ex., apresentação, conteúdo, funcionalidade, interação) com padrões e expectativas pessoais. Essa percepção do Usuário é avaliada utilizando uma escala de Likert de 7 pontos, por diferencial semântico avaliando aspectos hedônicos e pragmáticos.

Para cada aspecto, um grupo de sete escalas é utilizado, totalizando 28 pares de palavras: sete pares avaliam a Qualidade Pragmática ( $P Q)$, e outros sete, os Construtos de Atratividade/ Apelo (ATT), sendo considerados os aspectos pragmáticos.

Para a avaliação da qualidade hedônica, são apresentados catorze pares de palavras, subdivididos em: Qualidade Hedônica de Identidade (HQI) - que diz respeito a aspectos que possam auxiliar na forma como um indivíduo quer ser percebido pelos outros, comunicando sua identidade - e Qualidade Hedônica de Estímulo (HQS) - que dá suporte ao desenvolvimento pessoal do usuário, à melhoria de seus conhecimentos e habilidades (Hassenzhal et al., 2015; Hassenzhal et al., 2010).

Essa ferramenta tem sido usada amplamente em pesquisas em Design para avaliar tais requisitos sobre interação humano-computador. Segundo Padovani et al. (2012) esse era um dos métodos mais citados para pesquisa sobre experiência do usuário (UX) naquele momento, configurando sua popularidade e utilização no Brasil.

Faya (2018) em sua pesquisa sobre comércio eletrônico, verificou as traduções realizadas por Lira e Tavares (2015) e Cardoso (2013) e constatou algumas inconsistências. Entende-se que não é uma tradução simples, uma vez que a versão original do AttrakDiff2TM 2.0 é alemã, sendo encontradas também versões em inglês. procedeu então a tradução com a participação de um painel de especialistas trilíngues (português, inglês e alemão), conferindo a intenção de tradução dos autores da versão alemão para inglês e então, produzindo a versão em português. Essa tradução foi validada depois por um grupo de pesquisadores brasileiros. Só a partir dessa validação Faya procedeu à aplicação do AttrakDiff2TM 2.0 em sua pesquisa.

A título de curiosidade, apresenta-se na figura 1 as três versões do AttrakDiff2TM 2.0, em português, inglês e alemão.

\footnotetext{
${ }^{3}$ AttrakDiff2 ${ }^{\mathrm{TM}}$ 2.0. www.attrakdiff.de
} 
Figura 1: Versões inglês e português (proposta por Faya, 2018) do AttrakDiff2 ${ }^{\mathrm{TM}} 2.0$.
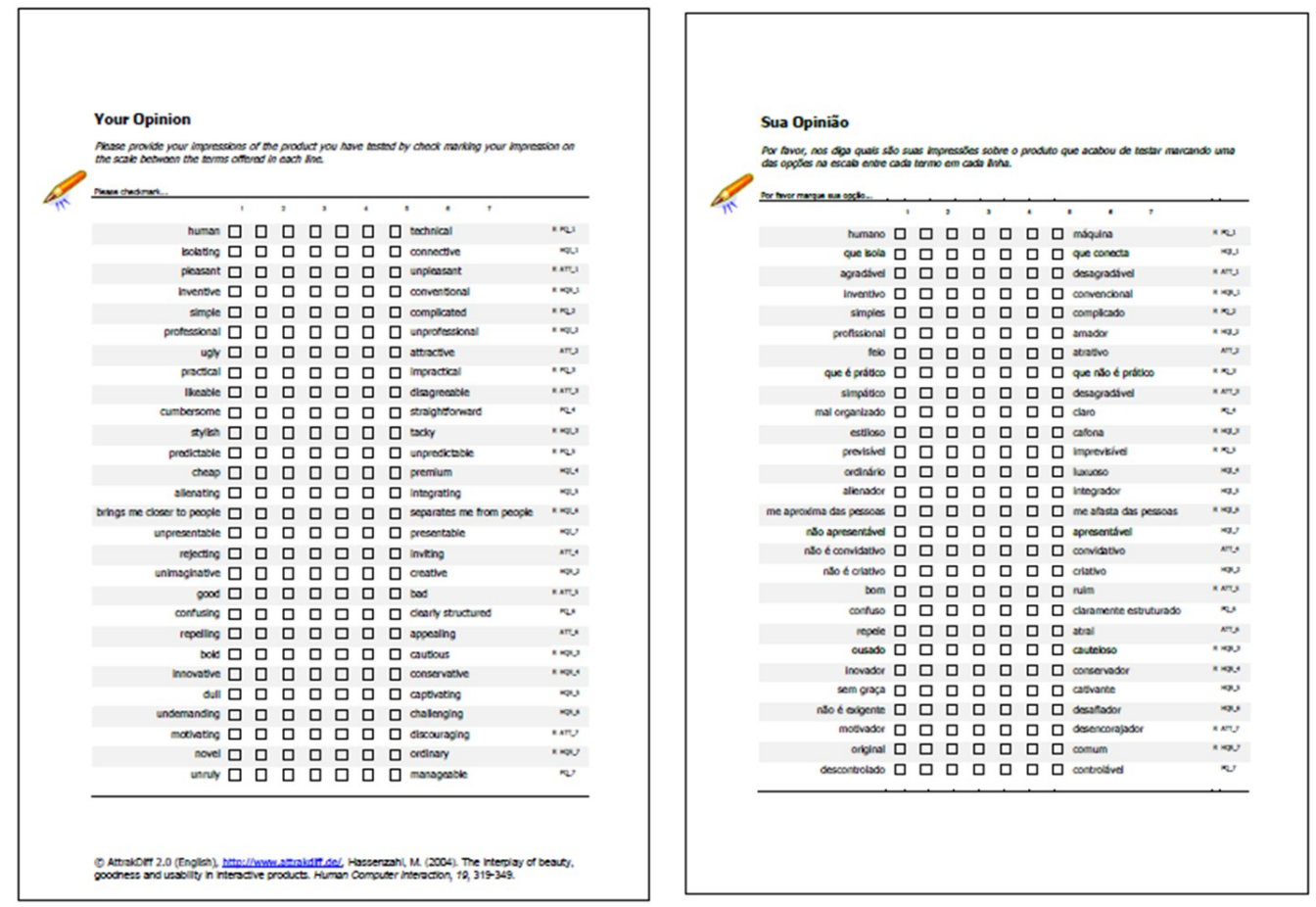

Ainda que todo o cuidado tenha sido tomado por Faya (2018) para chegar à melhor versão em português para os pares de palavras apresentadas, outros pesquisadores poderão ainda discordar.

\section{Exemplo 2: quando traduzir não é suficiente para ser compreensível}

O segundo caso aqui apresentado foi a tradução do questionário "Atitudes, percepções e crenças: uma pesquisa sobre segurança de tráfego", parte de uma pesquisa sobre design da informação também desenvolvida no âmbito do Design, realizada pelos mesmos autores desse artigo.

O questionário, originalmente desenvolvido em inglês (britânico) é dividido em seções para investigar as atitudes sobre o tráfego adotada por seus respondentes. Como outros questionários sobre comportamento do motorista (Driver Behaviour Questionnaire, DBQ), é dirigido não somente a motoristas, mas pedestres, ciclistas e demais condutores e atores do ambiente de tráfego.

Martinussen et al. (2013) afirma que "o questionário sobre comportamento do motorista é um dos instrumentos mais amplamente utilizados para a pesquisa autodeclarada. Ainda que os DBQs sejam muito populares, a sua aplicabilidade em grupos culturais distintos ainda precisa ser examinada."

Esse DBQ apresenta seções autodeclaradas de comportamento de pedestres sobre dados demográficos, opções de transporte e experiência, atitudes, percepções de risco, crenças, como descrito por Dinh et al. (2020), Mcllroy et al. (2019a, 2020), and Mcllroy et al. (2019b).

Três tradutores profissionais (com formação em Português/ Inglês) foram contatos para procederem conforme proposto por Brislin (1970), tendo a equipe de pesquisa como parceiros 
para discussão. Após algumas versões em português, a equipe verificou que somente o processo de tradução não seria suficiente, compreensível ou acessível para os respondentes em português. O principal ponto de discussão era a pertinência dos termos utilizados, sem que houvesse a mudança do que deveria ser questionado, sempre fiel à versão original.

Essa situação apontou para a necessidade de uma abordagem de linguagem simples, limitando-se não somente às questões linguísticas entre português e inglês, mas considerando também os aspectos culturais e de vida dos brasileiros, enfocando como cada questão seria perguntada e como seria apresentada a esses voluntários. Encontra-se aí, inclusive, uma clara vantagem da aplicação 'cara a cara' do questionário. Ainda que seja um formulário onde o pesquisador não pode interferir na interpretação, ele pode perceber imediatamente uma aparente confusão do entrevistado, podendo remediar isso no ato, ao sugerir sinônimos que facilitem a compreensão da pergunta, por exemplo.

Aqui se configura o projeto da informação: não se tratava de 'traduzir', mas de oferecer uma alternativa que fosse de encontro às demais características de informação necessárias para a compreensão do que era apresentado, perguntado. Nesse caso, configurado como informação escrita, que permitisse o processamento e decodificação do que era apresentado. Um exemplo foi a questão sobre religião, apresentada na Tabela 1. Ainda que a tradução fosse realizada de forma correta, outras opções precisaram ser incluídas para que os respondentes de identificassem com o que estava sendo perguntado e que permitissem sua escolha, refletindo os aspectos culturais dos respondentes.

Tabela 1: Adaptação das opções de resposta sobre religião na versão do questionário em português. As opções estão apresentadas na mesma ordem do DBQ

\begin{tabular}{ll}
\hline Versão original (inglês) & Conceito utilizado \\
\hline 1. No religion & 1. Sem religião \\
2. Buddhist & 2. Católico Romano \\
3. Christian & 3. Cristão (Metodista, Batista, Testemunha de Jeová, \\
4. Hindu & outros) \\
5. Jewish & 4. Espirita, Kardecista \\
6. Muslim & 5. Umbanda, Candomblé \\
7. Sikh & 6. Judeu \\
8. Any other religion & 7. Budista \\
& 8. Muçulmano \\
& 9. Hindu \\
& 10. Outra religião \\
\hline
\end{tabular}

O questionário chegou a seu formato final depois de três aplicações piloto. Na primeira, foi realizada com dez voluntários (oito respostas válidas, completas). As questões originais formuladas com expressões como 'mensalmente,' 'semanalmente,' ou 'anual' foram alteradas para 'diariamente.' Como exemplo, na pergunta 'indique sua renda anual bruta' foi substituída por 'Qual a SUA renda mensal? Considere o valor total recebido, antes dos descontos de impostos.' A questão anual não faria sentido, já que no Brasil os salários são mensais e a resposta original implicaria em um cálculo matemático. E o destaque para a palavra 'SUA' em maiúsculas, tinha intenção de chamar a atenção para a renda individual, e não familiar. 
O segundo teste piloto, que implementou as mudanças mencionadas considerou dez voluntários (e sete respostas válidas, completas). Ainda assim os respondentes comentaram que algumas questões apresentavam um tom pouco usual, muito formal e em algumas questões, isso provocava dúvida e confusão ao responder. Além disso, quando o questionário foi aplicado em pessoas de baixa escolaridade, em função do grande número de perguntas seria necessário explicar, reformular as questões para que fossem melhor entendidas, sugerindo novas mudanças. Outro aspecto foi em relação ao formulário utilizado, na plataforma Google forms. Trata-se de uma plataforma gratuita, que não permitia destaques de informações como uso de negrito ou cores, limitando o projeto da informação. Nesse momento, definiu-se a utilização da plataforma SurveyMonkey, para que esse aspecto do projeto da informação fosse solucionado. Essa mudança pode ser observada nas figuras 2, 3 e 4 .

Figura 2: Exemplo de escala de avaliação apresentada nas plataformas Google forms (esquerda) SurveyMonkey (direita)
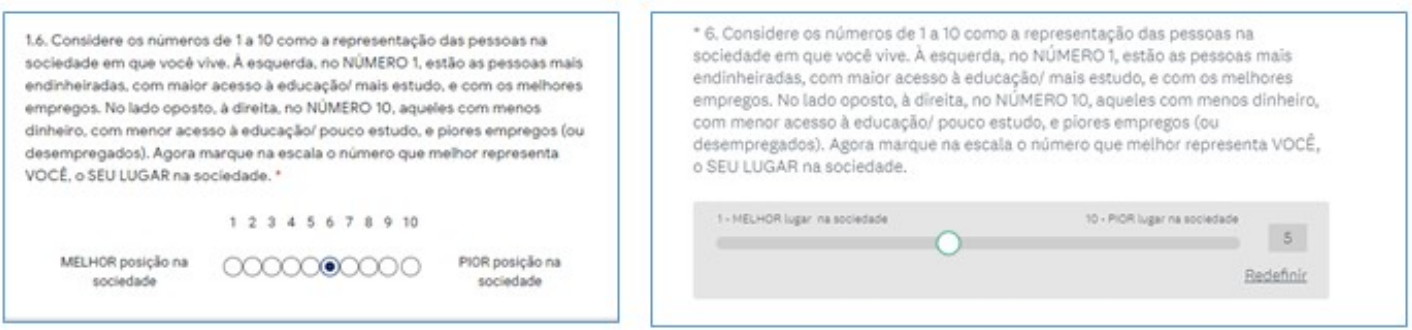

Figura 3: Exemplo de destaque de informações no texto apesentada nas plataformas Google forms (esquerda) SurveyMonkey (direita)

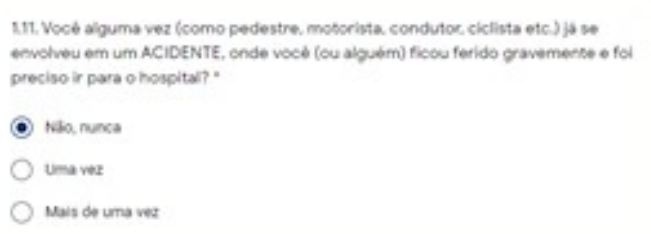

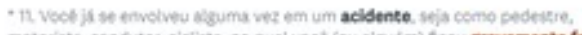
motorista, condutor, ciclista, no qual voce (ou alguém) ficou grwemente ferido fol preciso ir para o hospleat?

Mas de una ves

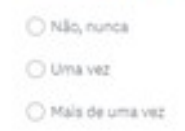

Figura 4: Exemplo de destaque de informações no texto introdutório apesentada nas plataformas Google forms (esquerda) SurveyMonkey (direita)
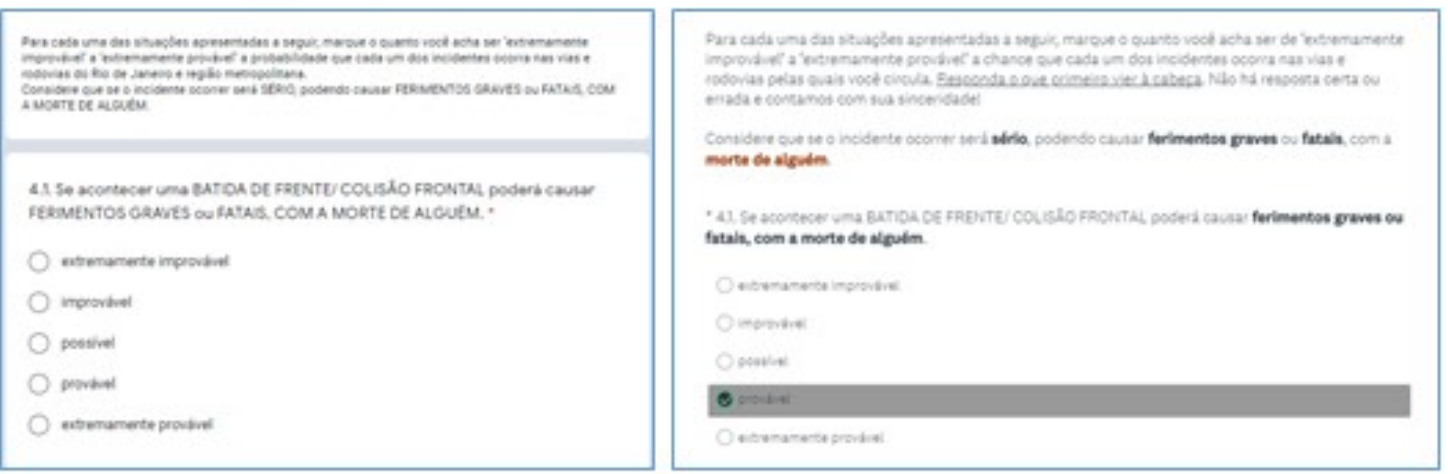
Uma abordagem de linguagem simples foi aplicada, com a reescrita de frases, levando à algumas modificações, alinhada à proposta de Waller $(2011$, p. 9):

Procuramos por um vocabulário ao qual o usuário (respondente) provavelmente já foi exposto - isso pode ser um julgamento de senso comum, mas pode ser determinado verificando a frequência da palavra em um corpus relevante (um corpus é uma coleção de textos digitalizados para este tipo de análise). Esse deve ser julgado em relação ao nível assumido de expertise do leitor.

Uma vez que essas questões foram modificadas, um terceiro momento de testagem foi necessário, e 25 voluntários (com todas as respostas válidas) foi possível chegar ao formato final do questionário, a ser aplicado com a uma amostra mais ampla.

\section{Considerações finais e próximos passos}

A primeira informação importante é que ao longo desse texto, vários trechos citados são traduções de nossa autoria, com equivalências formais e funcionais.

Traduzir ferramentas de pesquisa de forma adequada é uma questão ética com o autor da ferramenta original, e com os respondentes. Essa adequação configura-se, tanto em uma questão de tradução, quanto de projeto da informação, dada a necessidade de prover aos usuários/ respondentes a ferramenta tal qual ela foi proposta.

No primeiro exemplo apresentado, verificou-se que Faya (2018) propôs uma versão distinta as pesquisas por ela consultada, mostrando cuidado no processo de escolhas das palavras, voltando à versão original em alemão. Contudo, outros autores podem ter realizado outras versões em português para o AttrakDiff2TM 2.0 que diferem de sua proposta.

$\mathrm{Na}$ tradução do $\mathrm{DBQ}$, que além da tradução utilizou-se a abordagem a linguagem simples, o desafio para os autores foi ainda maior, uma vez que esse formulário apresentava 122 questões, e levava aproximadamente 30 a 40 minutos para ser respondido, completado. Cabe lembrar que o questionário foi um formulário apresentado pessoalmente aos respondentes e posteriormente, enviado online, uma vez que a pesquisa foi conduzida durante o momento de isolamento social. Nesse caso, o respondente não tem a presença do pesquisador para sanar suas dúvidas. Considerando essa participação remota, e o perfil dos respondentes, todas essas etapas foram necessárias para que os respondentes fossem alcançados e concluíssem o formulário até o final.

A partir dos exemplos apresentados, sugerem-se aqui alguns itens a serem observados no processo de tradução de ferramentas de pesquisa:

- Utilizar o método proposto por Brislin (1970). Mesmo que o pesquisador tenha sido alfabetizado em dois (ou mais) idiomas, incluindo a língua portuguesa, o ideal é proceder a tradução com o apoio de profissionais, objetivando a melhor versão possível para o nosso idioma;

- Nem sempre 'traduzir' para o português significa facilidade de compreensão ou acesso à informação. São necessários tantos testes pilotos quanto necessários, até que se atinja uma versão compatível com o público alvo de respondentes; 
- Os aspectos do design da informação são fundamentais para o destaque, visualização e compreensão das informações apresentadas. Aí inclui-se a linguagem simples, com a escolha de palavras e redação que não distorcem a versão original, mas adequam ao público respondente. No entanto, nem o design da informação, nem a abordagem da linguagem simples são itens triviais, que qualquer pessoa pode fazer. $O$ ideal é que a equipe que fará a tradução da ferramenta seja multidisciplinar.

Finalmente, deixamos a reflexão de Krznaric, filósofo, (2015, s/n), por entendermos que além do compromisso ético (já mencionado), trata-se de empatia com os voluntários da pesquisa:

O que descobri é que pessoas extremamente empáticas têm algo em comum. Elas se esforçam para cultivar seis hábitos - um conjunto de atitudes e práticas diárias que animam os conjuntos de circuitos empáticos em seus cérebros, permitindo-lhes compreender como outros veem o mundo. $O$ desafio que enfrentamos, se tivermos a esperança de realizar plenamente o Homo empathicus que reside dentro de cada um de nós (...) Abra os olhos para empatia e perceberá que ela está por toda parte, é a matéria em meio à qual nos movemos. Mas se é assim, qual é o problema? (...) Porque neste momento da história estamos sofrendo de um 'déficit de empatia' crônico, tanto na sociedade quanto em nossa vida pessoal.

\section{Agradecimento}

Os autores agradecem ao Conselho Nacional de Desenvolvimento Cientifico e Tecnológico (CNPq), à Coordenação de Aperfeiçoamento de Pessoal de Nível Superior - Brasil (CAPES código de financiamento 001) e à PUC-Rio pelo apoio e financiamento dessa pesquisa.

Os autores reconhecem, ainda, a contribuição dos profissionais de tradução envolvidos nos dois processos apresentados no artigo, bem como os voluntários dos testes. Sem a participação deles, não terias sido possível chegar à uma boa versão em português dos formulários apresentados como exemplos.

\section{Referências}

Web of Science (2020) Highly Cited Researchers. 2020 Recipients. https://recognition.webofscience.com/awards/highly-cited/2020/

Bell, R. T. (1991). Translation and translating: Theory and practice. London: Longman.

Brasil. Governo do Brasil (2020a). Matriz para análise de um texto - Linguagem Simples. 2 p. 2020. https://www.gov.br/pt-br/guia-de-edicao-de-servicos-do-gov.br/publicacoes-ecursos/matriz-para-analise-de-um-texto.pdf/.

CAPES Coordenação de Aperfeiçoamento de Pessoal de Nível Superior (2017) Plataforma Sucupira. Cursos avaliados e reconhecidos. Disponível em: https://sucupira.capes.gov.br/sucupira/public/consultas/coleta/programa/quantitativos/quantit ativoles.jsf?areaAvaliacao $=29 \&$ areaConhecimento $=61200000$

Cardoso, G. C. (2013) Avaliação da experiência do usuário durante o desenvolvimento de um aplicativo social móvel. [Dissertação de mestrado]. Design Gráfico. Universidade Federal de Santa Catarina. Brasil. 
Catford, F. C. (1980). Uma teoria lingüística da tradução. São Paulo: Cultrix.

De Souza, J. P. (1998) Teorias da tradução. Uma versão integrada. Ver. de Letras, n. 20, vol 1/ 2, jan/dez 1998.

Dinh, D. D. et al. (2020). Examining the roles of multidimensional fatalism on traffic safety attitudes and pedestrian behaviour. Safety Science, 124, [104587]. DOI: $10.1016 /$ j.ssci.2019.104587

Faya, C. M. B. A experiência do usuário (UX) em sítios de comércio eletrônico de vestuário: um estudo ergonômico. [Dissertação de mestrado]. Departamento de Artes \& Design. Pontifícia Universidade Católica do Rio de Janeiro. Brasil. 2018.

Hassenzahl, M. (2003) AttrakDiff: Ein Fragenbogen sur Messung wahrgenommener hedonischer und pragmatischer Qualitat. Mensch \& Computer. Interaktion in Bewegung, Stuttgart, 2003. pp. 187- 196.

Hassenzahl, M. (2004) The Interplay of Beauty, Goodness, and Usability in Interactive Products. Human-Computer Interaction, New York: Lawrence Erlbaum Associates Inc, vol 19, pp. 319-349.

Hassenzahl, M. et al (2015). Experience - oriented and Product-oriented evaluation: Psychological Need Fulfillment, Positive Affect, and Product Perception. International Journal of Human-Computer Interaction, n 31, pp. 530 - 544.

Hassenzahl, M.; Diefenbach, S.; Goritz, A. (2010). Needs, affect and interactive products: facets of user experience. Interacting with computers, v. 22, n. 5, pp. $353-362$.

Krznaric, R. (2015) O poder da empatia: A arte de se colocar no lugar do outro para transformar o mundo. Rio de Janeiro: Zahar. Ebook.

Lira, R. P. J; Tavares, T. (2015) Aprendendo pela interação: uma experiência com o uso de interfaces tangíveis e realidade aumentada voltada para curvas de níveis. Anais do $6^{\circ}$. Simpósio Hipertextos e Tecnologias na Educação e $2^{\circ}$ Colóquio internacional de Educação com Tecnologias. Recife/ PE, Brasil.

Martinussen, L. M. et al. (2013a) Age, gender, mileage and the DBQ: The validity of the Driver Behavior Questionnaire in different driver groups. Accident Analysis and Prevention, 52 (2013) 228- 236

Mcllroy, R. C. et al (2020). How do fatalistic beliefs affect the attitudes and pedestrian behaviours of road users in different countries? A cross-cultural study. Accident Analysis and Prevention. DOI: 10.1016/j.aap.2020.105491

Mcilroy, R. C. et al. (2019a). Exploring the relationships between pedestrian behaviours and traffic safety attitudes in six countries. Transportation Research Part F: Traffic Psychology and Behaviour. DOI: 10.1016/j.trf.2019.11.006

Mcllroy, R. C. et al. (2019b). Vulnerable road users in low-, middle-, and high-income countries: validation of a Pedestrian Behaviour Questionnaire. Accident Analysis and Prevention, 131, 80-94. DOI: 10.1016/j.aap.2019.05.027

Padovani, S. et al. (2012) Usabilidade e user experience, usabilidade versus user experience, usabilidade em user experience? Uma discussão teórico-metodológica sobre comunalidades e diferenças. $12^{\circ}$ Ergodesign \& USIHC. 12 Congresso internacional de Ergonomia e Usabilidade de Interfaces de Interfaces Humano-Computador. 
Penteado; A. C. B.; Ribeiro, R. P. (2016) Ferramentas para a avaliação de design emocional em projetos de design. UTFPR faz pesquisa em Design. Curitiba: UFTPR. Disponível em: https://utfprfazpesquisaemdesign.wordpress.com/2016/12/01/ferramentas-para-a-avaliacaode-design-emocional-em-projetos-de-design/

Plain Language Association International. (2021). What is plain language? Disponível em: http://plainlanguagenetwork.org

Redish, J. C. (2000) What is information design? Tech. Commun., vol. 47, no. 2, pp. 163-164.

Schriver, K. (2017): Plain Language In The US Gains Momentum: 1940-2015. IEEE Transactions on Professional Communication, vol. 60, n. 4, December 2017.

Waller, R. (2011) What makes a good document? The criteria we use. Simplification centre. Technical paper 2. Disponível em: https://www.simplificationcentre.org.uk/

\section{Sobre o(a/s) autor(a/es)}

Claudia Mont'Alvão, Dra., PUC-Rio, Brasil <cmontalvao@puc-rio.br> Carolina Esteves, graduanda em Design, PUC-Rio, Brasil <kikaspaulino@hotmail.com> Mariana Furtado Dias, graduanda em Design, PUC-Rio, Brasil <marianafdias@icloud.com> 